\section{Robot Path Planning in Uncertain Environments: A Language-Measure- Theoretic Approach}

\author{
Devesh K. Jha \\ Mechanical \& Nuclear Engineering Department \\ Pennsylvania State University, \\ University Park, PA 16802 \\ e-mail: dkj5042@psu.edu
}

\section{Yue Li}

Mechanical \& Nuclear Engineering Department

Pennsylvania State University,

University Park, PA 16802

e-mail: yol5214@psu.edu

\section{Thomas A. Wettergren}

Naval Undersea Warfare Center,

Newport, RI 02841;

Mechanical \& Nuclear Engineering Department

Pennsylvania State University,

University Park, PA 16802

e-mail: t.a.wettergren@ieee.org

\author{
Asok Ray \\ Fellow ASME \\ Mechanical \& Nuclear Engineering Department \\ Pennsylvania State University, \\ University Park, PA 16802 \\ e-mail: axr2@psu.edu
}

This paper addresses the problem of goal-directed robot path planning in the presence of uncertainties that are induced by bounded environmental disturbances and actuation errors. The offline infinite-horizon optimal plan is locally updated by online finite-horizon adaptive replanning upon observation of unexpected events (e.g., detection of unanticipated obstacles). The underlying theory is developed as an extension of a grid-based path planning algorithm, called $\boldsymbol{v}^{\star}$, which was formulated in the framework of probabilistic finite state automata (PFSA) and language measure from a control-theoretic perspective. The proposed concept has been validated on a simulation test bed that is constructed upon a model of typical autonomous underwater vehicles (AUVs) in the presence of uncertainties. [DOI: 10.1115/1.4027876]

Keywords: path planning, language measure, probabilistic finite state automata

\section{Motivation and Introduction}

In general, path planning of robots (e.g., AUVs) and unmanned aerial vehicles (UAVs)) aims to optimize either travel time, energy usage, or safety of operations. Recently, much work has been reported for path planning in the presence of environmental uncertainties. A few examples follow.

Garau et al. [1] used the $A^{\star}$ algorithm for path planning of AUVs by taking the effects of ocean currents into consideration.

Contributed by the Dynamic Systems Division of ASME for publication in the Journal of Dynamic Systems, Measurement, and Control. Manuscript received January 23, 2014; final manuscript received May 31, 2014; published online October 21, 2014. Assoc. Editor: Jongeun Choi.

This material is declared a work of the U.S. Government and is not subject to copyright protection in the United States. Approved for public release; distribution is unlimited.
Pêtrès et al. [2] reported path planning of AUVs, in which a fast marching algorithm was used to model the effects of ocean currents as an anisotropic cost function. Rhoads et al. [3] solved a dynamic Hamilton Jacobi Bellman equation to obtain the optimal "time-to-go" with a discontinuous and dynamic cost function for path planning in the presence of ocean currents. Recently, Lolla et al. [4] developed a methodology for AUV path planning, in which the concept of flow advection was combined with nominal vehicle motion until the desired goal was reached. Majumdar and Tedrake [5] generated libraries in the offline precomputation stage for online robust motion planning, where some of the library members were regenerated for collision avoidance. Blackmore et al. [6] designed a chance-constrained predictive stochastic controller to ensure that the probability of failure (e.g., collision) is less than a specified threshold even in the presence of randomly varying uncertainties. Chakravorty and Kumar [7] used the concepts of probabilistic roadmaps and rapidly exploring random trees [8] to construct feedback controllers for robust planning in the presence of motion uncertainties. However, in many path planning algorithms reported in literature, the offline computation does not take into account potential runtime constraints (e.g., unanticipated obstacles), and online adaptation is computationally infeasible.

Robots (e.g., AUVs) are expected to operate in environments with external disturbances and hence, considering the effects of these disturbances is critical for mission success. Consequently, the algorithms of path planning must be capable of real-time execution on in situ computational platforms. To meet these challenges, Chattopadhyay et al. [9] formulated the $v^{\star}$ algorithm in the framework of PFSA from a control-theoretic perspective. The $v^{\star}$ algorithm performs robot path planning for a specified goal by optimizing the language measure of the PFSA model $[10,11]$ that represents the workspace for the robot. In this setting, optimal path planning is equivalent to maximization of a PFSA's performance, based on a quantitative measure of probabilistic regular languages. This concept of robot path planning jointly maximizes the probability of reaching the target and the probability of staying clear of the obstacles. Such a notion of goal-directed safe (e.g., Pareto optimization [12] of reaching the goal and collision avoidance) navigation of autonomous vehicles is particularly important in the context of planning in the presence of disturbances, especially to ensure collision-free navigation. In this case, Markov decision process (MDP) tools may not be suitable because of the requirement of robust adaptation in real time [5]. The situation becomes worse for lack of observability, because the formulation of a partially observable MDP would become computationally intractable for a real-time solution.

Language-measure-theoretic path planning offers an inherent advantage of global monotonicity [9] in the sense that the solution iterations are finite and that a sequence of final waypoints is generated. The PFSA, constructed out of the robot's workspace, is optimized via an iterative sequence of combinatorial operations to maximize the language measure vector elementwise. Nevertheless, the $v^{\star}$ algorithm is different from a conventional search algorithm in the sense that it automatically generates a measure gradient (e.g., no potential function required), which is maximized at the goal. Furthermore, the time complexity of each iteration step is linear relative to the problem size (e.g., the dimension of the discretized state space), and computation of the measure vector in a distributed fashion makes the algorithm especially suitable for high-dimensional planning. These advantages render the presented method potentially suitable for (offline) planning and (online) adaptation in the presence of uncertainties.

As an extension of the $\boldsymbol{v}^{\star}$ algorithm [9], this paper formulates an algorithm of grid-based robot path planning, which has been validated on a simulation test bed of AUVs in the presence of uncertain ocean currents and actuation errors. Major contributions of the paper beyond the work on the $\boldsymbol{v}^{\star}$ algorithm, reported by Chattopadhyay et al. [9], are outlined below:

- The notion of uncertainty is associated with the uncontrollable transitions in a controlled autonomous system. This 
concept is different from the common practice of superimposing the effects of disturbances on the planned trajectory of a robotic system.

- The offline infinite-horizon optimal plan is locally updated by online finite-horizon adaptive replanning upon observation of unexpected events (e.g., detection of unanticipated obstacles).

\section{Brief Review of Language Measure Theory}

This section summarizes the concept of (signed real) language measure [10] of PFSA [13] and the role of language measure for optimal control [11] of PFSA. While the theories of language measure and the associated optimal control are developed in Refs. [10] and [11], this section introduces pertinent definitions and summarizes the essential concepts that are used in the sequel.

DeFINITION 2.1. (Alphabet and Word) An alphabet $\Sigma$ is a nonempty finite set of symbols. A finite-length string of symbols from $\Sigma$ is called a word and the empty word (i.e., a string of no symbols) is denoted as $\epsilon$. A word $w$ of $\ell$ symbols has length $|w|=\ell$ and $|\epsilon|=0$. The set of all words (including $\epsilon$ ) is denoted as $\Sigma^{\star}$; the set of all words of length $\ell$ is denoted as $\Sigma^{\ell}$; and the set of all infinite strings of symbols is denoted as $\Sigma^{\omega}$.

Referring to Definition 2.1, the cardinalities of the following sets are noted below:

- $|\Sigma| \in \mathbb{N}$, where $\mathbb{N}$ is the set of positive integers.

- $\left|\Sigma^{\ell}\right|=|\Sigma|^{\ell}$ for any positive integer $\ell$.

- $\left|\Sigma^{\star}\right|=|\mathbb{N}|$, i.e., $\Sigma^{\star}$ is countably infinite $\left(\aleph_{0}\right)$.

- $\left|\Sigma^{\omega}\right|=|\mathbb{R}|$, i.e., $\Sigma^{\omega}$ is uncountably infinite (continuum).

Definition 2.2 (Prefix and Suffix) Let $\Sigma$ be an alphabet. The operation of concatenation of two words is closed on $\Sigma^{\star}$, i.e., if $w=x y$, then $w \in \Sigma^{\star} \forall x, y \in \Sigma^{\star}$; and $\epsilon$ is the identity element of the concatenation monoid [14]. In that case, $x$ is called a prefix of $w$, and $y$ is called a suffix of $w$.

DEFINITION 2.3. A PFSA over an alphabet $\Sigma$ is a quintuple

$$
\mathbb{G} \triangleq(Q, \Sigma, \delta, \pi, \chi) \text {, where }
$$

- $Q$ is the nonempty finite set of states, i.e., $|Q| \in \mathbb{N}$;

- $\delta: Q \times \Sigma^{\star} \rightarrow Q$ is the transition map that satisfies the following conditions: $\forall q \in Q \forall s \in \Sigma \forall w \in \Sigma^{\star}$ $\delta(q, \epsilon)=q ;$ and $\delta(q, w s)=\delta(\delta(q, w), s)$.

- $\pi: Q \times \Sigma^{\star} \rightarrow[0,1]$ is the morph function of state-specific symbol generation probabilities, which satisfies the following conditions: $\forall q \in Q \forall s \in \Sigma \forall w \in \Sigma^{\star}$

$\pi(q, s) \geq 0 ; \sum_{s \in \Sigma} \pi(q, s)=1 ;$ and $\pi(q, \epsilon)=1 ; \pi(q, w s)=\pi(q, w) \times \pi(\delta(q, w), s)$.

- $\chi: Q \rightarrow[-1,1]^{|Q|}$ is the vector-valued characteristic function that assigns a signed (normalized) real weight to each state.

The $(|Q| \times|Q|)$ state transition probability matrix $\boldsymbol{P}$ is defined as

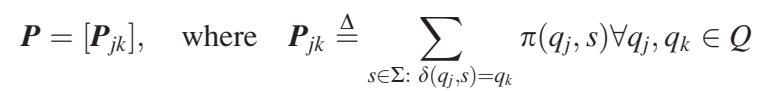

Note: $\boldsymbol{P}$ is a non-negative stochastic matrix [15].

Remark 2.1. The restrictions $\left.\delta\right|_{Q \times \Sigma} \rightarrow Q$ and $\left.\pi\right|_{Q \times \Sigma} \rightarrow[0,1]$ of the functions $\delta$ and $\pi$ in Definition 2.3 can be directly obtained from the local structure of the PFSA. The unrestricted functions $\delta$ and $\pi$ can then be computed over the entire domain by using the recursive relations listed in Definition 2.3. Physical interpretations of $\chi$ are briefly provided in Subsection 2.1, while the details are reported in Definition 5 of Ref. [10].

Definition 2.4. (Language) Let $(Q, \Sigma, \delta, \pi, \chi)$ be a PFSA. The language generated by all words, which terminates at a state $q_{k} \in Q$ after starting from $q_{j} \in Q$, is defined as

$$
L\left(q_{j}, q_{k}\right) \triangleq\left\{w \in \Sigma^{*}: \delta\left(q_{j}, w\right)=q_{k}\right\}
$$

The language generated by all words, which may terminate at any state $q \in Q$ after starting from $q_{j} \in Q$, is defined as

$$
L\left(q_{j}\right) \triangleq \bigcup_{q \in Q} L\left(q_{j}, q\right)
$$

Given a PFSA $(Q, \Sigma, \delta, \pi, \boldsymbol{\chi})$, the following facts are considered before the notion of a measure is introduced on the language $L$ (see Definition 2.4).

- The language $L$ is an at most countable (i.e., finite or countably infinite) set because $L \subseteq \Sigma^{\star}$. Therefore, the power set $2^{L}$ is either finite or uncountable; if uncountable, the cardinality of $2^{L}$ is the same as that of $\mathbb{R}$ or that of the standard Borel algebra $\mathcal{B}(\mathbb{R})[16]$.

- A measurable space $\left(L, \Sigma^{\omega} 2^{L}\right)$ can be constructed where every singleton set of a semi-infinite string of symbols, whose suffix is a word in $L$, is a measurable set $[10,16]$. For brevity, the $\sigma$-algebra $\Sigma^{\omega} 2^{L}$ is denoted as $2^{L}$, because every word $w \in L$ is prefixed by a semi-infinite string of symbols. That is, $w$ represents $x w$ and $\{w\} \in 2^{L}$ implies $\{x w\} \in \Sigma^{\omega} 2^{L}$, where $x \in \Sigma^{\omega}$ is arbitrary.

DefinItion 2.5. (Language Measure) Let $L\left(q_{j}, q_{k}\right)$ and $L\left(q_{j}\right)$ be languages on a PFSA $(Q, \Sigma, \delta, \pi, \chi)$ and let $\theta \in(0,1)$ be a parameter. A signed real measure $\mu_{\theta}^{j k}: 2^{L\left(q_{j}, q_{k}\right)} \rightarrow \mathbb{R}$ (that satisfies the requisite axioms of measure [16]) is defined as

$$
\mu_{\theta}^{j k}\left(L\left(q_{j}, q_{k}\right)\right) \triangleq \sum_{w \in L\left(q_{j}, q_{k}\right)} \theta(1-\theta)^{|w|} \pi\left(q_{j}, w\right) \chi\left(q_{k}\right)
$$

The measure of the language $L\left(q_{j}\right)$ is defined as

$$
\nu_{\theta}^{j}\left(L\left(q_{j}\right)\right) \triangleq \sum_{q_{k} \in Q} \mu_{\theta}^{j k}\left(L\left(q_{j}, q_{k}\right)\right)
$$

Following Definition 2.5, the set of language measures for a PFSA is interpreted as a real-valued vector of dimension $|Q|$ and is denoted as $\boldsymbol{v}_{\theta}$ whose $j$ th component is $\nu_{\theta}^{j} \in \mathbb{R}$ corresponding to the state $q_{j} \in Q$. Following Theorem 1 in Ref. [10], the language measure of the PFSA $(Q, \Sigma, \delta, \pi, \chi)$ in Eq. (2.5) is expressed vectorially as

$$
\boldsymbol{v}_{\theta}=\theta[\mathbb{I}-(1-\theta) \mathbf{P}]^{-1} \boldsymbol{\chi}
$$

where $\mathbf{P}$ is the state transition matrix (see Definition 2.3) and the inverse on the right side exists for all $\theta \in(0,1)$. Furthermore, as $\theta \rightarrow 0^{+}$, the matrix $\theta[\mathbb{I}-(1-\theta) \mathbf{P}]^{-1}$ converges to the Cesaro matrix $\mathcal{P} \triangleq \lim _{k \rightarrow \infty} \frac{1}{k} \sum_{j=0}^{k-1} \mathbf{P}^{j}$. Then, the limiting measure vector $\boldsymbol{v}_{0}$ is obtained as [11]

$$
\boldsymbol{v}_{0} \triangleq \lim _{\theta \rightarrow 0^{+}} \boldsymbol{v}_{\theta}=\lim _{\theta \rightarrow 0^{+}} \theta[\mathbb{I}-(1-\theta) \mathbf{P}]^{-1} \boldsymbol{\chi}=\mathcal{P} \boldsymbol{\chi}
$$

where $\mathbb{I}$ is the $(|Q| \times|Q|)$ identity matrix. 
2.1 Control Perspectives. From the perspectives of discreteevent optimal control [11], the limiting language measure vector $v_{0}$ in Eq. (6) is physically interpreted as follows. The language measure of a symbol string starting at a state $q_{j} \in Q$ is interpreted to be the product of the morph probability (conditioned on the state $q_{j}$ ) and the characteristic weight $\chi_{k}$ of the terminating state $q_{k}$. For example, if $q_{k}$ is a desirable state (e.g., goal) to terminate, then $\chi_{k}$ should be assigned to be positive; similarly, $\chi_{k}$ should be negative for an undesirable terminating state (e.g., obstacle) $q_{k}$. Thus, the characteristic weights are assigned to represent the control specifications (i.e., larger positive weights to more desirable states) and the language measure represents the goodness of the particular string relative to the given specification and the PFSA model [10]. In the sense of Definition 2.5, the limiting language measure $\nu_{0}^{j}$ sums up to the limiting measures of each string starting from state $q_{j}$. Thus, $\nu_{0}^{j}$ captures the goodness of $q_{j}$ based on not only its own characteristic weight but also on how good are the strings that will be generated from $q_{j}$ in the future. In essence, the language measure provides a quantitative comparison of viable control policies [11].

Definition 2.6. (Control philosophy) Let $q_{j} \stackrel{s}{\longrightarrow} q_{k}$ be the state transition under occurrence of the event $s$. If the event $s$ is disabled at $q_{j}$ by a supervisory action, then this state transition from $q_{j}$ to $q_{k}$ will be prevented by forcing the plant to stay at the original state $q_{j}$. Thus, disabling an event $s$ at a given state $q$ results in deletion of the original transition and appearance of the self-loop $\delta(q, s)=q$ with the probability of occurrence of $s$ at state $q$ remaining unchanged in the supervised plant and also in the unsupervised plant.

Definition 2.7. (Controllable transitions) For a given plant, transitions that can be disabled in the sense of Definition 2.6 are defined to be controllable; a transition that is not controllable is called uncontrollable. The set of controllable transitions in a plant is denoted as $\mathbf{C}$.

If the supervisor is allowed to disable all subsets (including the empty set $\emptyset$ and the set $\mathbf{C}$ itself) of the set $\mathbf{C}$ of controllable transitions, then there exists a bijection between the set of all possible supervision policies and the power set $2^{\mathrm{C}}$; in other words, there are $2^{|\mathbf{C}|}$ possible supervisors and each supervisor is uniquely identifiable with a subset of $\mathbf{C}$. Thus, the language measure $\boldsymbol{v}$ allows a quantitative comparison of different policies.

Definition 2.8. (Controlled PFSA and optimal measure $\boldsymbol{v}^{\star}$ ) An optimally controlled (or supervised) PFSA $\mathbb{S} \triangleq(Q, \Sigma, \delta, \pi, \chi, \mathbf{C})$ is a sextuple that maximizes its language measure $\boldsymbol{v}_{0}$ by disabling a selected subset of $\mathbf{C}$. The optimized measure $\boldsymbol{v}_{0}$ is denoted as $\boldsymbol{v}^{\star}$.

\section{Description of the Simulation Test Bed}

This section describes the simulation test bed that is built upon a rigid-body dynamic model of the planar motion of a generic AUV [17]. The rationale for adopting such a simple model is that the usage of the proposed language-measure-theoretic concepts can be unambiguously presented to explain how to control the AUV motion in the presence of environmental disturbances and actuation errors. Nevertheless, the theory is applicable on a dynamically feasible graph with any degree of details.

The model takes into account the effects of yaw $(\psi)$ and the velocity vector of surge rate $(u)$, sway rate $(v)$, and yaw rate $(r)$, i.e., $\mathbf{v} \triangleq\left[\begin{array}{lll}u & v & r\end{array}\right]^{\mathrm{T}}$; but the effects of heave $(z)$, roll $(\phi)$ and pitch $(\theta)$ are assumed to be negligible [17]. The resulting equations of motion are obtained as

$$
\mathbf{M} \dot{\mathbf{v}}+\mathbf{C}(\mathbf{v}) \mathbf{v}+\mathbf{D}(\mathbf{v}) \mathbf{v}=\tau \quad \text { and } \quad \dot{\psi}=r
$$

where $\mathbf{M}=\mathbf{M}^{\mathrm{T}}$ is the inertia matrix; $\mathbf{C}(\mathbf{v})=-\mathbf{C}(\mathbf{v})^{\mathrm{T}}$ is the Coriolis centripetal matrix; $\mathbf{D}(\mathbf{v})$ is the damping matrix; and $\tau \triangleq\left[\begin{array}{lll}\tau_{u} & \tau_{v} & \tau_{r}\end{array}\right]^{\mathrm{T}}$ is the vector of the system inputs that represent the forces generated by the on-board actuators as well as those produced by environmental disturbances (e.g., ocean current). In this model, control actions are generated as functions of the surge rate $(u)$ and yaw rate $(r)$ only, i.e., the vehicle control input is restricted to be $\tau_{\text {con }}=\left[\begin{array}{lll}\tau_{u} & 0 & \tau_{r}\end{array}\right]^{\mathrm{T}}$. Surge and sway components of the ocean current vector $\mathbf{v}_{\mathrm{c}}$ are modeled in terms of its direction $\beta$ and yaw angle $\psi$ as

$$
u_{c}=\left|\mathbf{v}_{\mathrm{c}}\right| \cos (\beta-\psi) \text { and } v_{c}=\left|\mathbf{v}_{\mathrm{c}}\right| \sin (\beta-\psi)
$$

Assuming that the vehicle's on-board controller maintains the desired yaw angle $\psi$ during its course of motion (i.e., letting $\dot{\psi}=r \equiv 0$ ), the dynamic motion model is further simplified to obtain the travel distance $\mathbf{F}$ and $\mathbf{G}$ along the surge and sway directions [17], respectively, as

$$
\mathbf{F}\left(\tau_{u}, u_{c}\right) \triangleq \int_{t_{0}}^{t_{f}} u(t) \mathrm{d} t \quad \text { and } \quad \mathbf{G}\left(v_{c}\right) \triangleq \int_{t_{0}}^{t_{f}} v(t) \mathrm{d} t
$$

\section{Problem Formulation}

This section formulates the problem of path planning for planar motion of robots in the presence of uncertainties induced by environmental disturbances, where the workspace is partitioned as a finite number of cells. Major assumptions in the problem formulation are listed below:

(1) Partial knowledge of the static obstacles.

(2) No moving obstacles.

(3) Partitioning of the workspace into cells as a regular grid.

(4) Bounded disturbances and actuation errors with the errors of PFSA states being limited to neighboring cells of the origin cell.

Depending on the resolution of planning, a cell may represent a single location or a subregion in the planning environment. The neighborhood of a cell is defined by taking into account feasible dynamics of transitions to that cell.

The robot motion is modeled as controllable transitions among the cells as the robot chooses to execute its moves; such moves may depend on the fidelity of motion discretization and the robot's intrinsic dynamics. Based on the specifications of intercell transitions, each cell in the partitioned workspace is modeled as a PFSA state, where controllable transitions are defined by the corresponding state transition map.

Let a PFSA $\mathbb{G}_{\mathrm{NAV}}=(Q, \Sigma, \delta, \pi, \boldsymbol{\chi})$ (see Definition 2.3) represent the navigation model of a robot, where the states in $Q$ are cell locations in the original workspace and the alphabet size $|\Sigma|$ is determined from the number of events that may cause controllable transitions. For simplicity of exposition, this paper considers a planar robot that is allowed to freely rotate about its geometric center, implying that $|\Sigma|=9$. As an example, let the robot be in the state $q$ (see Fig. 1) and then let the robot make a choice to move to one of the states labeled 1 through 8 or it may stay at $q$. While the state transition map $\delta$ (restricted to $Q \times \Sigma$ ) is constructed in the neighborhood of a PFSA state and the morph function $\pi$ (restricted to $Q \times \Sigma$ ) are constructed under the condition that, with negligible state estimation error, the robot can choose any controllable transition to execute at a cell. Hence, all controllable transitions at a cell are assumed to be equally likely for the unsupervised automaton $\mathbb{G}_{\mathrm{NAV}}$. The vector-valued characteristic function $\chi$ (see Definition 2.3) is chosen based on a priori known goodness of individual states, explained in Subsection 2.1. Since the environment map could be only partially known, the robot must adapt to the unforeseen obstacles in real time.

Uncertain (e.g., fault-induced and environmental) disturbances may cause uncontrollable transitions in the robot motion as seen in the example of Fig. 1, where the robot may erroneously move to an unplanned state, thus interfering with control actions of robot path planning (see Assumption (4) at the beginning of this section). Therefore, probabilistic decisions of path planning need 
to be made based on the set of the available controllable actions of the robot to mitigate the effects of these disturbances. In this model, the state transition probabilities serve as a discretized representation of the effects of disturbances on the robot dynamics, where the transition probability $\mathbb{P}\left(q_{j} \mid q_{i}, s\right)$ represents the probability of reaching state $q_{j}$ after executing an action $s$ from state $q_{i}$. Using the simplified model developed in the last section, the vehicle's relative position in the next time step (from the previous position after it makes a move $s \in \Sigma$ in the global Cartesian coordinate frame) is expressed as

$$
\begin{aligned}
& \Delta x=\mathbf{F} \cos (\psi)-\mathbf{G} \sin (\psi) \\
& \Delta y=\mathbf{F} \sin (\psi)+\mathbf{G} \cos (\psi)
\end{aligned}
$$

The corresponding transition probabilities $\mathbb{P}$ under the specified simplifying assumptions are then computed in terms of the probabilities $\mathcal{P}$ as

$$
\mathbb{P}\left(q_{j} \mid q_{i}, s\right)=\mathcal{P}\left([\Delta x, \Delta y] \in q_{j} \mid q_{i}, s\right)
$$

In the absence of disturbances, $\mathbb{P}\left(q_{j} \mid q_{i}, s\right)$ is either 0 or 1 such that $\mathbb{P}\left(\delta\left(q_{i}, s\right) \mid q_{i}, s\right)=1$ and $\mathbb{P}\left(q_{j} \mid q_{i}, s\right)=0 \forall q_{j} \in Q \backslash \delta\left(q_{i}, s\right)$.

While the PFSA $\mathbb{G}_{\mathrm{NAV}}$ specifies the discretized motion of the planar robot, the matrix $\mathbb{P}$ contains information about the effects of the disturbances on its motion. With the knowledge of $\mathbb{G}_{N A V}$ and $\mathbb{P}$, the path planning problem is reduced to the optimal supervision problem of $\mathbb{G}_{\text {NAV }}$ (in the sense of Definition 2.8) in the presence of disturbances specified by $\mathbb{P}$. Detection of unforeseen obstacles during the robot operation may result in a change of the characteristic weight vector $\chi$. In this context, new obstacles are considered as undesirable terminating states and, therefore, are assigned negative characteristic weights (see Subsection 2.1).

\section{Solution Approach}

Formulation of the path planning problem as a PFSA-based navigation allows computation of optimally feasible paths via the language-measure-theoretic optimization scheme. For the unsupervised model, the robot is free to choose from any of the defined controllable events from any cell in the workspace.

The optimization algorithm, presented as Algorithm 1, selectively disables controllable transitions to ensure that the measure vector of the navigation automaton is elementwise maximized. Selected controllable transitions are disabled to optimize the state transition probability matrix $\mathbf{P}$ (see Definition 2.3 ). This is accomplished by maximizing the limiting measure vector (see Eq. (6)) in the sense of Definition 2.8, which corresponds to the optimally supervised PFSA model for the robot. This implies that the supervised robot is constrained only to choose among the enabled moves at each state such that a Pareto trade-off is achieved between minimization of the collision probability and simultaneous maximization of the goal-reaching probability. An important point to note is that even though the measure values are based on optimization of a PFSA, an optimal and feasible plan is obtained that can be executed in a deterministic sense.

For planning in the presence of environmental disturbances, it is critical to consider the robot's vulnerability to uncertainties created by such disturbances (see Fig. 1). In the work presented in Refs. [9] and [11], the measure vector was optimized by ignoring the uncontrollable transitions arising from the uncertainties. However, ignoring the uncontrollable transitions in the presence of disturbances could be fatal for autonomous robots. In this paper, the effects of the uncontrollable transitions are taken into consideration while optimizing the measure vector of the navigation automaton. In this framework, a combinatorial sequence of enabling and disabling of transitions optimizes a weighted combination of minimizing the probability of collision and maximizing the probability of reaching the goal in the presence of known environmental disturbances. Depending on the strength of environmental

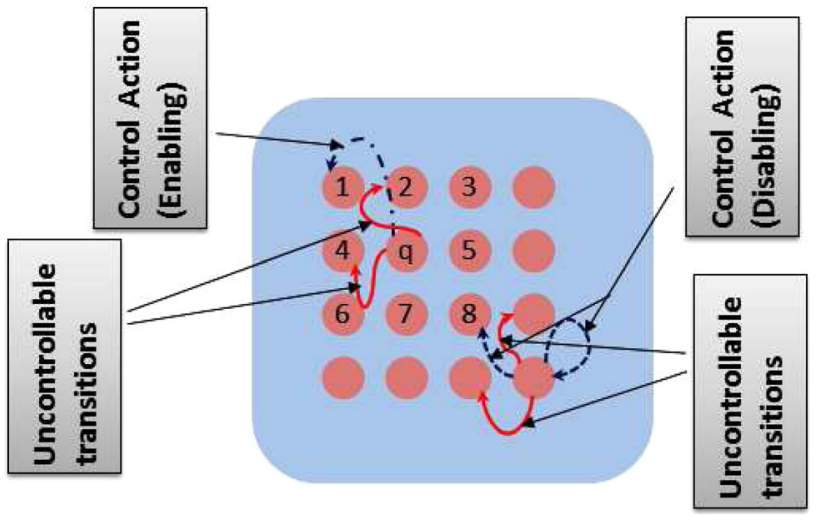

Fig. 1 Illustration of the effects of uncontrollable transitions due to the disturbances. It is shown how uncontrollable transitions may cause the robot to end up in a different neighboring state of the origin cell, thus interfering with control actions.

disturbances, the controller attempts to make an optimal trade-off between performance (i.e., path length) and robustness to uncertainties.

With Monte Carlo simulation of the disturbances on the test bed, conditional probabilities for the robot (i.e., AUV) to terminate at a neighboring state $q_{j}$ are generated as

$$
\mathbb{P}\left(q_{j} \mid q_{i}, s\right) \text { such that } \sum_{q_{j} \in N\left(q_{i}\right)} \mathbb{P}\left(q_{j} \mid q_{i}, s\right)=1
$$

where $q_{j} \in Q$ is a neighboring state of $q_{i}$ and $s \in \Sigma$ represents the control action.

Let $\mathbb{G}_{\mathrm{NAV}}=(Q, \Sigma, \delta, \pi, \boldsymbol{\chi})$ be the unsupervised navigation automaton. From the knowledge of the morph probability $\pi$ and the conditional probability distribution $\mathbb{P}$ due to the environmental disturbances, a family of probability matrices, $\mathbf{P}_{G}(\cdot, \cdot, j)$, $j=1,2, \cdots,\left|N\left(q_{i}\right)\right|$ is constructed, where $\left|N\left(q_{i}\right)\right|$ is cardinality of the set $N\left(q_{i}\right)$ of neighborhood states (including itself) of the state $q_{i}$ (e.g., $\left|N\left(q_{i}\right)\right|=9$ for any internal state of a planar robot), defined as

$$
\mathbf{P}_{\mathbb{G}}\left(q_{i}, s, q_{j}\right) \triangleq \mathbb{P}\left(q_{j} \mid q_{i}, s\right) \times \pi\left(q_{i}, s\right) \quad \forall q_{i} \in Q
$$

where $s \in \Sigma$ is a control action and $\delta\left(q_{i}, s\right)=q_{j}$ is a neighboring state (including itself) of $q_{i} . v_{\theta}^{N\left(q_{i}\right)}$ (used in line 24 of Algorithm 1) is a local measure vector of state $q_{i}$ and consists of the measure values of the states $q_{j} \in N\left(q_{i}\right)$. The transition matrix $\mathbf{P}_{\mathbb{G}}$ is optimized in an iterative fashion by Algorithm 1 such that the strings, which are likely to lead to collision in the presence of the disturbances, are disabled. At the same time, feasible paths to the goal are retained so that the measure vector is elementwise maximized.

For path planning in a large-dimensional state space, a distributed iterative scheme is recommended for the computation of the language measure, which was reported in an earlier publication [18]. The algorithm of optimized language measure vector is presented as Algorithm 1.

Salient properties of Algorithm 1 are delineated below:

- Computation of the uncertain behavior of the robot's motion caused by environmental disturbances: The sequence of disabling and enabling actions at the robot's discretion is generated by (elementwise) maximization of the language measure vector. To relax the assumption of restriction of uncontrollable transitions to the neighboring states, a probability distribution over the entire workspace could be considered instead of using a local measure vector in line 24 of Algorithm 1. This is also true in trajectory planning for 


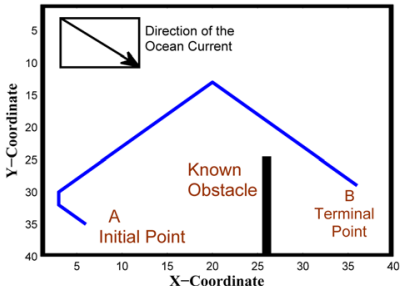

(a)

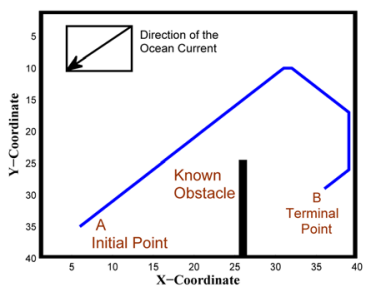

(b)

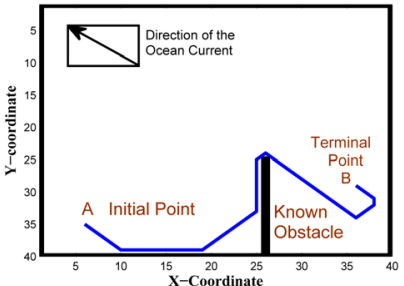

(c)

Fig. 2 Optimal paths under different directions of the ocean current. (The vehicle is constrained to stay in the box and not hit the walls.)

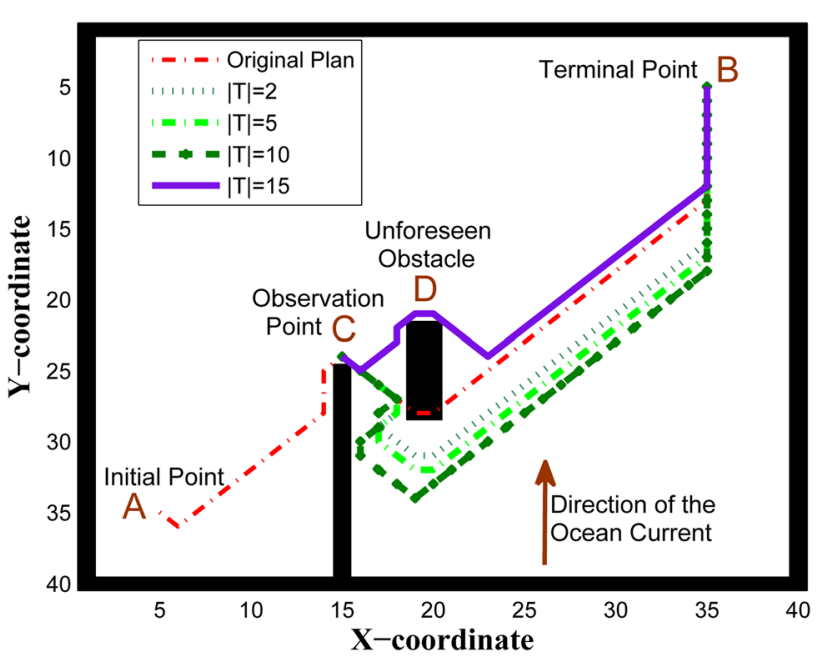

Fig. 3 Real-time replanning over a finite time horizon $T$

UAVs, where the environmental disturbances may require widening of its flight envelope.

- Generation of the optimized measure vector $\boldsymbol{v}^{\star}$ for robot navigation based on its PFSA model, along with the associated optimized outputs $\pi^{\star}$ and $\boldsymbol{P}_{\mathbb{G}}^{\star}$. The path to the goal from arbitrary initial locations is obtained by following the gradient of the measure vector.

Real-time obstacle avoidance in the presence of disturbances is a difficult problem due to the disturbance-induced uncontrollable transitions. An online replanning algorithm must propagate the motion model in time and make sure that the robot is clear of the obstacle. In this regard, one may take advantage of the fact that the stochastic navigation matrix (i.e., the optimized state transition matrix of the controlled PFSA) contains the information on the disturbances. To make a local adaptation, the original infinitehorizon measure vector is augmented with the information on recently discovered obstacles. The effects of this obstacle are considered on a finite-time horizon of replanning by using the already optimized stochastic navigation matrix $\mathbf{P}_{\mathbb{G}}^{\star}$. However, as the stochastic matrix was already optimized by considering the effects of disturbances, the replanning keeps the vehicle clear of the obstacle under such disturbances. The quality of adaptation is expected to improve with an increase in the replanning time horizon. Hence, a choice of the replanning time horizon $(T)$ depends on the trade-off between the computation time and quality of the solution. The steps involved in replanning are succinctly presented in Algorithm 2 , which efficiently computes a feasible path. This is accomplished by removing those strings that reach the goal via the newly detected obstacle states from the language generated by any state of the PFSA. The measure vector is consequently updated by removing those strings from the original optimal language generated by the states of the PFSA, i.e., by subtracting the measure of such strings (see Definition 2.5) from the original language measure. The resulting path improves monotonically in the replanning time horizon because the estimate of the measure of such strings improves monotonically.

Algorithm 1. Distributed updating of measure vector for planning in the presence of disturbances

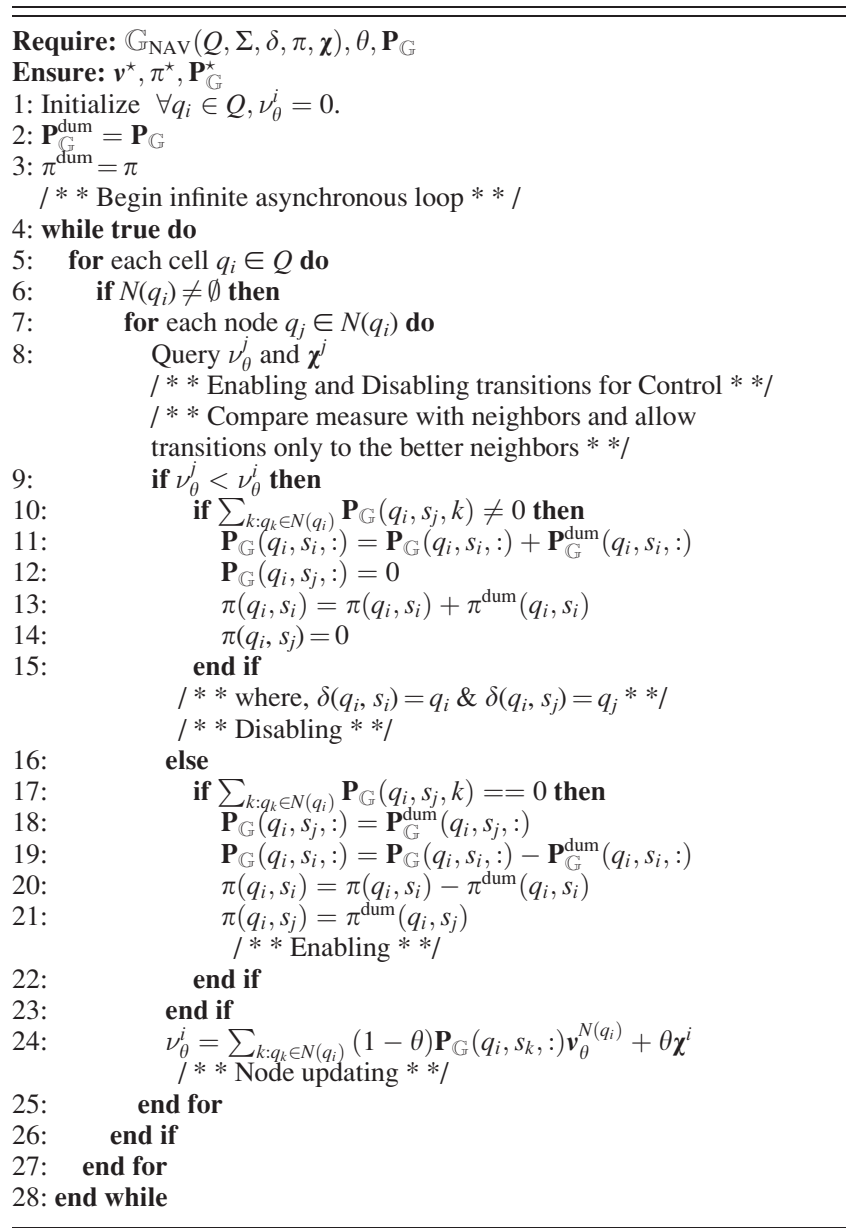

Algorithm 2. Updating of measure vector for replanning

Require: $\mathbb{G}_{\mathrm{NAV}}\left(Q, \Sigma, \delta, \pi^{\star}, \boldsymbol{\chi}\right), \theta, \mathbf{P}_{\mathbb{G}}^{\star}, \boldsymbol{v}^{\star},|T|$

Ensure: $v_{\text {replan }}^{\star}, \mathbf{P}_{\mathbb{G}, \text { replan }}^{\star}, \pi_{\text {replan }}^{\star}$

1: Update $\chi$ with new information about obstacles

2: Initialize $\boldsymbol{v}_{\theta}=\boldsymbol{v}^{\star} \& n=1$

3: $\mathbf{P}_{G}^{\text {dum }}=\mathbf{P}_{G}^{\star}$ and $\pi^{d u m}=\pi^{\star}$

4: while $n \leq|T|$ do

5: $\quad$ Lines 4 through 24 of Algorithm 1

6: $n=n+1$

7: end while

Remark 5.1. The time complexity of Algorithm 1 is $\tau_{1} \sim \mathbf{O}\left(|Q||N(q)|^{2}\right)$, for a PFSA with $|Q|$ states and $|N(q)|$ is the 


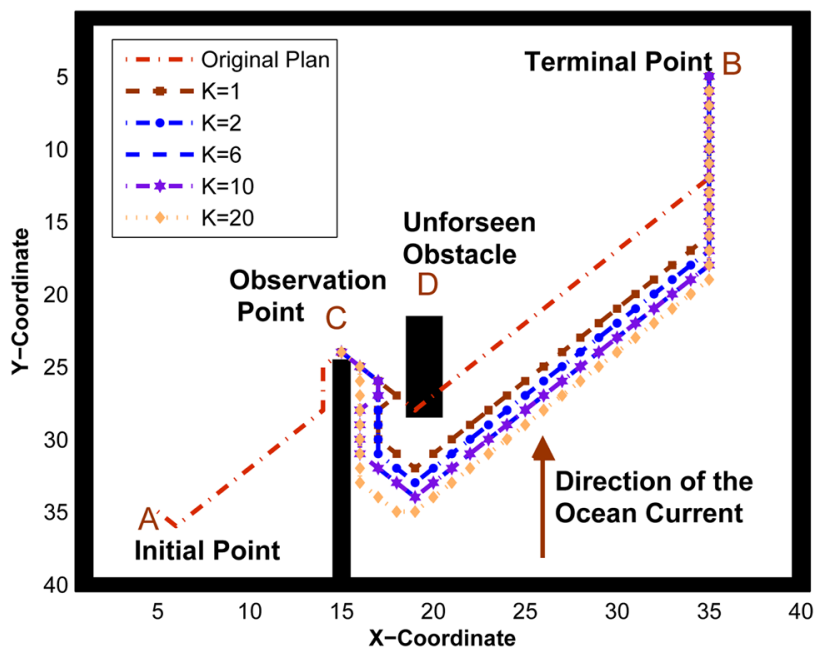

Fig. 4 Replanning with $|\boldsymbol{T}|=2$ for different characteristic weights

maximum neighborhood size of the PFSA states [18]. The time complexity of Algorithm 2 is $\tau_{2} \sim \mathbf{O}(|T||N(q)|)$, where $T$ is the finite-time horizon of replanning.

\section{Interpretation of the Results}

This section presents pertinent results of AUV simulation for the proposed method of path planning in the presence of uncertainties due to ocean currents. To justify the usage of a disturbance-aware path planning algorithm for AUVs and to demonstrate the algorithm's efficacy to react to different types of disturbances, Fig. 2 shows the (simulated) optimal paths of an AUV that is navigated from point $A$ to point $B$ in a similar environment with three different directions of the ocean current. The optimal nominal paths in these three cases are significantly different, because the paths are calculated to accommodate the disturbances due to the ocean current.

During actual navigation, the AUV is likely to deviate from the nominal trajectory; however, it finds a path from any arbitrary state to the terminal state by following the gradient of the optimized measure vector. The nominal trajectory in each of the three plates in Fig. 2 shows the shortest safe path based on the expected deviations due to the disturbances.

Figure 3 presents a scenario, where the environment is only partially known a priori and an unforeseen obstacle at point $D$ is observed when the AUV reaches point $C$. Consequently, the original plan (i.e., without the knowledge of the obstacle at $D$ ) is altered to avoid a potential collision. In Fig. 3, the uncontrollable transitions (that are due to the ocean current) tend to push the vehicle upstream and path replanning attempts to bypass the obstacle at $D$; however, the amount of time available for online replanning is limited. Algorithm 2 is iteratively executed to improve the replanning monotonically with an increase in the span $|T|$ of time horizon $T$. As seen for $|T|=2,5$, and 10 in Fig. 3 , the respective replans are generated to navigate the AUV further down so that even if the vehicle is drifted upwards by the ocean current, it would still be able to avoid a collision with the obstacle. By increasing the replanning time horizon from $|T|=2$ to $|T|=10$, the plan becomes more robust to collision as it attempts to keep the vehicle clear of the obstacle by a larger distance by optimizing the margin of error due to the ocean currentinduced disturbances. A small replanning time horizon results in local greedy search of the configuration space; it limits the effects of the new obstacle to a narrow region around it. A smaller replanning time horizon also limits the expected deviation of the vehicle due to disturbances.

A global optimal policy could be significantly different from a local greedy solution obtained in limited time as seen in Fig. 3, where the replanned path for $|T|=15$ is different from those for $|T|=2,5$, and 10. While Algorithm 2 makes local perturbations in the initial plan to keep the AUV clear of an observed obstacle at $D$, a sufficiently large replanning time horizon (e.g., $|T|=15$ in Fig. 3) generates an optimal path such that the local changes in the replanned path may finally cause convergence to a global optimal path.

As the original optimal stochastic navigation matrix contains information of the disturbance, even a small replanning time horizon could keep the AUV clear of the obstacle in the presence of disturbances; however, robustness of the plans improve monotonically with increase in the replanning time horizon parameter $|T|$. In essence, a choice of $|T|$ depends on the in situ computational capabilities of the AUV and the time available for online updating of the path plan, which may depend on the AUV's speed and observation window.

For time-critical operations with limited computational capabilities, a goal of replanning is to ensure safety while maintaining an accepted level of performance (e.g., path length). This trade-off could be achieved in the language-measure-theoretic framework by selecting the characteristic weight of new-obstacle states during replanning, where an increased penalty on collision with an obstacle would enhance safety at the expense of a longer deviation from the original plan. This is achieved by having the characteristic weights of the newly observed obstacles states as $K \star \chi_{\text {obs }}$, where the multiplicative constant $K$ is a positive real parameter, while all other characteristic weights remain unchanged. Figure 4 shows the effects of changing the characteristic weight vector for the case $|T|=2$ in Fig. 3. As seen in Fig. 4, the safety margin is enhanced by increasing $K$, which is capable of accommodating larger anomalous behavior of the robot in the presence of ocean currents at the expense of an increased path length. For a family of admissible values of $K$, the convex hull of the generated solutions would lead to a Pareto-optimal front representing trade-off between safety and performance. For any particular real-time operation, the choice of the parameter $K$ is dependent on computational capabilities and time available for replanning as well as strength of disturbances.

\section{Summary, Conclusions, and Future Work}

This paper presents a generalized framework for robust path planning of AUVs in the presence of environmental disturbances and actuation errors. Concepts of recently developed language measure-based optimization $[10,11]$ have been applied to demonstrate successful navigation of an autonomous robot moving in an uncertain and partially known environment. The algorithm is robust to both modeling and environmental uncertainties; however, these uncertainties must be bounded. The efficacy of the proposed concept is demonstrated by numerical simulation on a test bed of an AUV moving in the presence of ocean currents and unforeseen obstacles. The paper is summarized below:

1. It presents a path planning philosophy that is fundamentally different from the ones reported in literature.

2. It performs online adaptation of the planning algorithms to unanticipated obstacles in the presence of disturbances.

Many path planning algorithms, reported in the current literature, may not be able to efficiently handle collision avoidance in real time in the presence of disturbances, primarily due to overwhelming computational requirements. It is shown in this paper through numerical simulations that the language-measure-theoretic algorithm can handle unanticipated obstacles in real time in the presence of disturbances. However, actual performance comparisons with state-of-the-art planning are yet to be demonstrated by experimental validation.

While there are numerous research areas for robot path planning based on language-measure-theoretic concepts, the following topics are recommended for future research. 
1. Construction of a biased sampling scheme while updating the language measure of nodes in a distributed fashion to enhance the quality of path planning and to mitigate the computational cost. Such a scheme is important for a tradeoff between exploration and exploitation.

2. Extension of the proposed method of robot path planning under time constraints as well as by minimizing the energy consumption.

3. Validation of the proposed method by laboratory experimentation on a networked robotic test bed with nonholonomic constraints [8].

4. Performance comparison with other methods like those presented in Refs. [5-7].

\section{Acknowledgment}

The authors acknowledge the benefits of discussion with Dr. Shashi Phoha at Applied Research Laboratory of Pennsylvania State University.

This work was supported in part by the U.S. Army Research Laboratory and the U.S. Army Research Office under Grant No. W911NF-13-11-0461 and by the U.S. Air Force Office of Scientific Research (AFOSR) under Grant No. FA9550-12-10270. Any opinions, findings, and conclusions or recommendations expressed in this publication are those of the authors and do not necessarily reflect the views of the sponsoring agencies.

\section{References}

[1] Garau, B., Alvarez, A., and Oliver, G., 2005, "Path Planning of Autonomous Underwater Vehicles in Current Fields With Complex Spatial Variability: An A* Approach," IEEE International Conference on Robotics and Automation (ICRA), Barcelona, Spain, April 18-22, pp. 194-198.

[2] Pêtrès, C., Pailhas, Y., Patrón, P., Petillot, Y., Evans, J., and Lane, D., 2007. "Path Planning for Autonomous Underwater Vehicles," IEEE Trans. Rob. Autom., 23(2), pp. 331-341.
[3] Rhoads, B., Mezić, I., and Poje, A., 2010, "Minimum Time Feedback Control of Autonomous Underwater Vehicles," IEEE Conference on Decision and Control (CDC), Atlanta, GA, Dec. 15-17, pp. 5828-5834.

[4] Lolla, T., Ueckermann, P., Yiğit, K., Haley, P. J. Jr., and Lermusiaux, P. F. J., 2012, "Path Planning in Time Dependent Flow Fields Using Level Set Methods," IEEE International Conference on Robotics and Automation (ICRA), St. Paul, MN, May 14-18, pp. 166-173.

[5] Majumdar, A., and Tedrake, R., 2013, "Robust Online Motion Planning With Regions of Finite Time Invariance," Algorithmic Foundations of Robotics X, Springer, Berlin, Germany, pp. 543-558.

[6] Blackmore, L., Ono, M., Bektassov, A., and Williams, B. C., 2010, "A Probabilistic Particle-Control Approximation of Chance-Constrained Stochastic Predictive Control," IEEE Trans. Rob., 26(3), pp. 502-517.

[7] Chakravorty, S., and Kumar, S., 2011, "Generalized Sampling-Based Motion Planners," IEEE Trans. Syst. Man Cybern. Part B Cybern., 41(3), pp. 855-866.

[8] LaValle, S. M., 2006, Planning Algorithms, Cambridge University, Cambridge, UK.

[9] Chattopadhyay, I., Mallapragada, G., and Ray, A., 2009, “ $\nu^{*}$ : A Robot Path Planning Algorithm Based on Renormalized Measure of Probabilistic Regular Languages," Int. J. Control., 82(5), pp. 849-867.

[10] Ray, A., 2005, "Signed Real Measure of Regular Languages for Discrete-Event Supervisory Control," Int. J. Control., 78(12), pp. 949-967.

[11] Chattopadhyay, I., and Ray, A., 2007, "Language-Measure-Theoretic Optimal Control of Probabilistic Finite-State Systems," Int. J. Control., 80(8), pp. 1271-1290.

[12] Miettinen, K. M., 1999, Nonlinear Multiobjective Optimization, Kluwer Academic Publishers, Boston, MA

[13] Ray, A., 2004, "Symbolic Dynamic Analysis of Complex Systems for Anomaly Detection," Signal Process., 84(7), pp. 1115-1130.

[14] Gill, A., 1976, Applied Algebra for the Computer Sciences, Prentice-Hall, Englewood Cliffs, NJ.

[15] Bapat, R. B., and Raghavan, T. E. S., 1997, Non-negative Matrices and Applications, Cambridge University, Cambridge, UK.

[16] Rudin, W., 1988, Real and Complex Analysis, 3rd ed., McGraw Hill, New York.

[17] Fossen, T. I., 1994, Guidance and Control of Ocean Vehicles, John Wiley, Chichester, West Sussex, UK.

[18] Chattopadhyay, I., and Ray, A., 2011, "GODDeS: Globally $\epsilon$-Optimal Routing Via Distributed Decision-Theoretic Self-Organization,” American Control Conference, San Francisco, CA, June 29-July 1, pp. 3215-3220. 